DIW BERLIN

Discussion

Papers

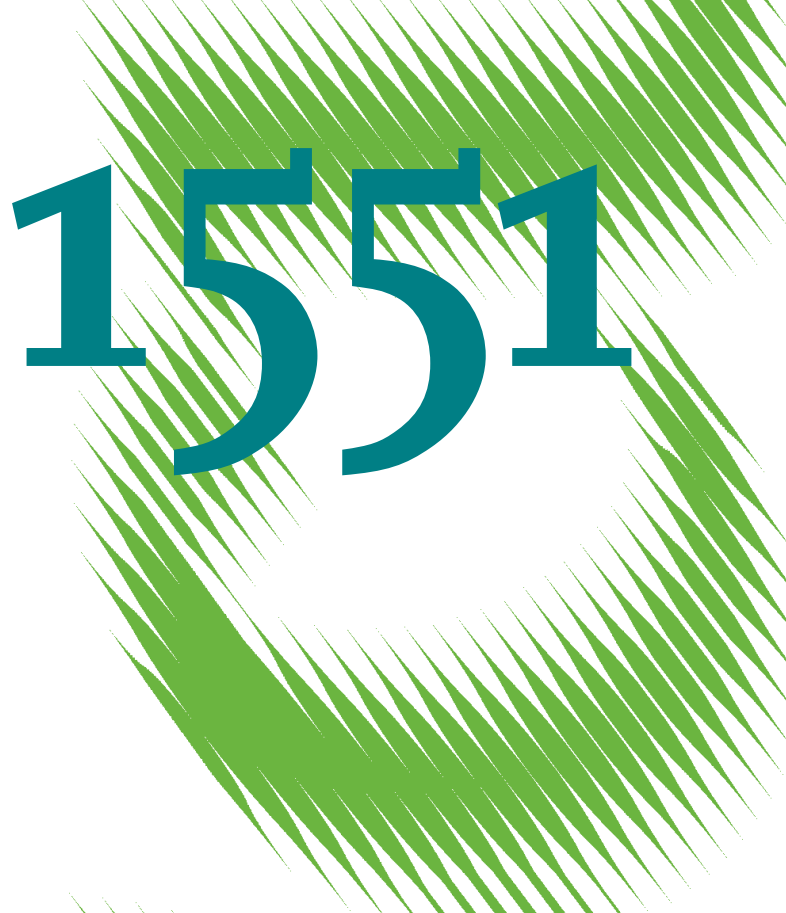

Coordinating Cross-Country

Congestion Management 
Opinions expressed in this paper are those of the author(s) and do not necessarily reflect views of the institute.

IMPRESSUM

(C) DIW Berlin, 2016

DIW Berlin

German Institute for Economic Research

Mohrenstr. 58

10117 Berlin

Tel. +49 (30) $89789-0$

Fax +49 (30) $89789-200$

http://www.diw.de

ISSN electronic edition 1619-4535

Papers can be downloaded free of charge from the DIW Berlin website:

http://www.diw.de/discussionpapers

Discussion Papers of DIW Berlin are indexed in RePEc and SSRN:

http://ideas.repec.org/s/diw/diwwpp.html

http://www.ssrn.com/link/DIW-Berlin-German-Inst-Econ-Res.html 


\title{
Coordinating Cross-Country Congestion Management
}

\author{
Friedrich Kunz ${ }^{*}$ \& Alexander Zerrahn ${ }^{*+}$
}

\begin{abstract}
$\underline{\text { Abstract }}$
We employ a detailed two-stage model to simulate the operation of the Central Eastern European electricity market and network. Implementing different cases of coordination in congestion management between national transmission system operators, numerical results show the beneficial impact of closer cooperation. Specific steps comprise the sharing of network and dispatch information, cross-border counter-trading, and multilateral redispatch in a flowbased congestion management framework. Efficiency gains are accompanied by distributional effects. Closer economic cooperation becomes especially relevant against the background of changing spatial generation patterns, deeper international integration of national systems, and spillovers of national developments to adjacent systems.
\end{abstract}

Keywords: $\quad$ Electricity, congestion management, network modeling, Europe

JEL-code: $\quad$ C63, L51, L94

\footnotetext{
${ }^{*}$ German Institute for Economic Research (DIW Berlin), Mohrenstr. 58, D-10117 Berlin, Germany.

${ }^{\dagger}$ Corresponding author: Alexander Zerrahn, DIW Berlin, Mohrenstr. 58, D-10117 Berlin, Germany; phone: +49 (0)30 89789453 , fax: +49 (0)3089789113; email: azerrahn@diw.de.
} 


\section{INTRODUCTION}

European electricity systems experience a fundamental change toward a low carbon infrastructure. By 2013, the variable renewables wind and solar accounted for $10 \%$ of electricity generation in the EU 28 , compared to $2 \%$ in 2005 (Eurostat, 2015). In the future, the renewables share is supposed to rise further in order to achieve the EU's ambitious climate goals (EC, 2011). At the same time, the electricity transmission system evolved when large-scale generation and national self-sufficiency was still the norm. The result, for instance for Germany, is a high number of hours with insufficient line capacities in the high voltage grid and increasing costs for congestion management (BNetzA and BKartA, 2012, 2013, 2014). In the long run, the transmission system can be recast to meet the new requirements. In the short run, network congestion must be relieved by counter-trading or redispatch measures. In both cases, the responsible entity, in most cases the Transmission System Operator (TSO), arranges an increase in generation at one end of the congested grid area, compensated by a decrease at the other end. The costs of these deviations from the spot market dispatch are socialized through network tariffs.

Physical flows in the electricity network cannot be directed, but spread according to Kirchhoff's laws. The resulting flows are, as such, not bound by national borders, but may put pressure on the operation of adjacent electricity systems. In Germany, for example, wind generation and lignite plants are concentrated in the north-eastern regions, whereas load centers are in the south and west. A share of the resulting electricity flows, so-called loop flows, is also transported through neighboring countries, for instance Poland and the Czech Republic, to re-enter Germany in Bavaria (BNetzA and BKartA, 2015). See Figure 1 for a stylized representation of our study region. These spillovers on adjacent transmission systems may pose new challenges for their operation ${ }^{3}$. These inter-dependencies highlight the relevance of international cooperation and call for coordinated measures of congestion management. In principle, the delineation of bidding zones, in which the network topology is economically not taken into account, can internalize congestion issues (for an analysis of the German case, see Egerer et al., 2015), with the limiting

\footnotetext{
${ }^{3}$ See, for example, THEMA (2013) and ČEPS et al (2013).
} 
case of nodal pricing. Irrespective of market areas, however, coordination issues between countries or zones remain to be resolved.

Figure 1. Stylized representation of the study region

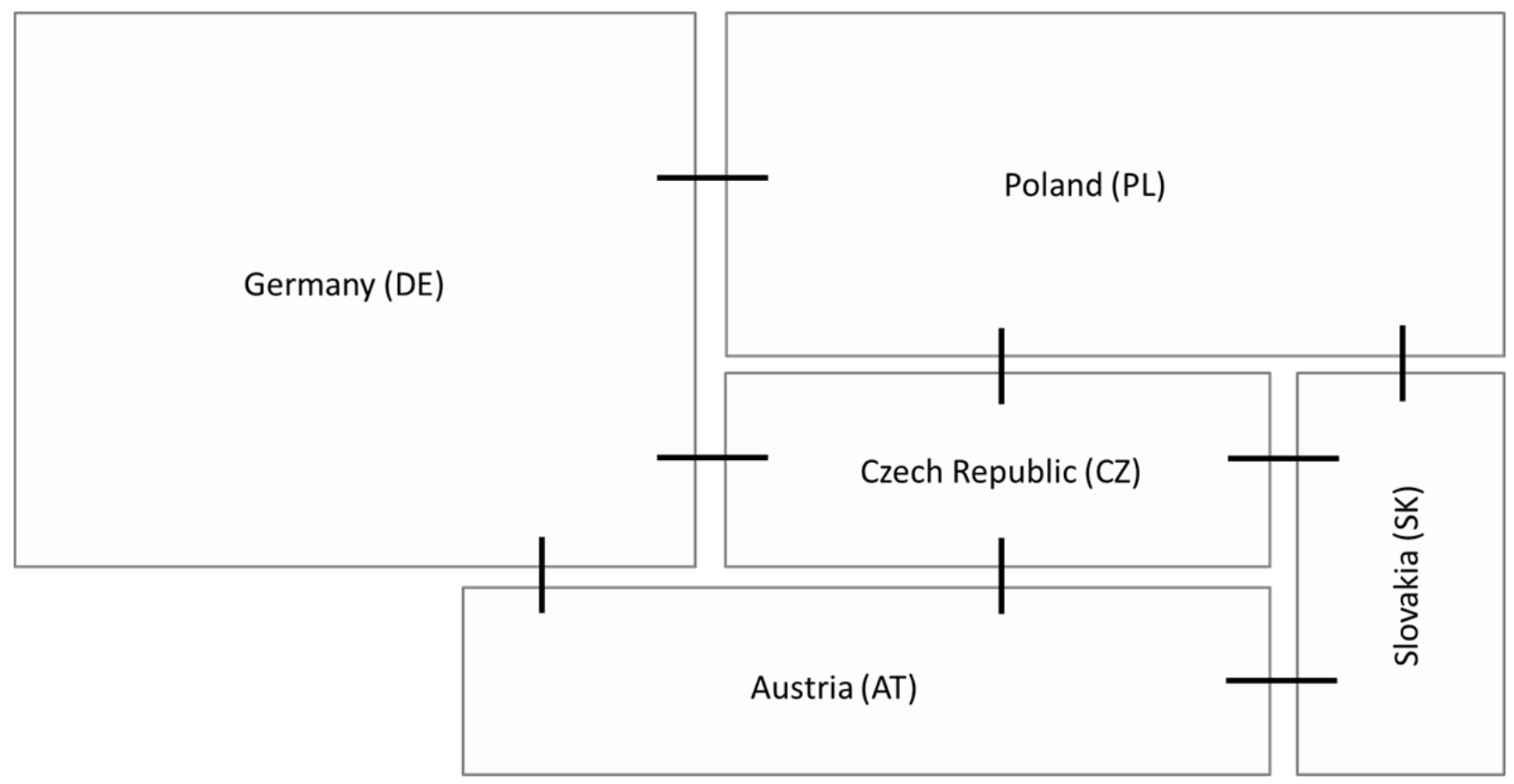

New guidelines for coordination are, in fact, on the rise: the European Commission (EC) envisages a European Energy Union to enhance economic efficiency, sustainability and security of supply. A vital part is the deeper integration of electricity markets through greater interconnectivity and closer cooperation in planning and operation of grids (EC, 2015a). Already within the Third Energy Package in 2009, so-called Network Codes were proposed, in order to harmonize cross-border trade and operation of adjacent electricity systems (EC, 2009). Specifically, the Network Code on Capacity Allocation and Congestion Management (CACM), enacted in 2015, requires TSOs to elaborate a common set of practices for alleviating congestion of cross-border relevance and coordinate on measures taken (EC, 2015b). Particularly, TSOs are urged to

abstain from unilateral or uncoordinated redispatching and countertrading measures of crossborder relevance. Each TSO shall coordinate the use of redispatching and countertrading resources taking into account their impact on operational security and economic efficiency. (EC, 2015b, p L197/53) 
Concerning the economic treatment of cross-border coordination, the literature identified cost savings from a transition to a full-fledged nodal pricing system, incorporating all network constraints in the market clearing process (Kunz, 2012; Neuhoff et al., 2013). Chaves-Avila et al. (2014) analyze that increased coordination, taking account of cross-zonal balancing issues in Germany, can reduce flawed incentives toward undue arbitrage behavior. More generally, Brunekreeft (2015) discusses efficiency gains from less fragmentation along the electricity value chain in Europe. From a methodological point of view, the coordination congestion management is analyzed by Oggioni et al. (2012). The authors develop a Generalized Nash Equilibrium (GNE) model to disentangle the responsibility of relieving line overflows between different players. Applications in Oggioni and Smeers (2012) and Oggioni and Smeers (2013) put forward that a higher degree of coordination can increase redispatch efficiency. Kunz and Zerrahn (2015) further elaborate on welfare gains from coordinating congestion management. They show that shared responsibility between German TSOs has the potential to substantially reduce costs by access to cheaper units.

While the bulk of the literature either focuses on intra-national congestion management, or addresses international issues from the perspective of integrating network constraints into market clearing, there is little academic evidence on cross-border coordination of congestion management. This paper fills that gap. We provide an in-depth analysis detailing cases of coordination, and highlighting the benefits of different instances of increased cooperation. To this end, we set up a two-stage model of the electricity system in our study region, comprising Austria (AT), the Czech Republic (CZ), Germany (DE), Poland (PL), and Slovakia (SK). The short-run stochastic unit commitment and dispatch model stELMOD (Abrell and Kunz, 2015) creates a market dispatch and determines the resulting physical electricity flows. Extending the work by Kunz and Zerrahn (2015), we implement different cases of coordination between national TSOs, and isolate the benefits of closer cooperation. In this respect, we vary the scope of national responsibilities, the balancing area, and the implicit auction mechanism for cross-border capacities.

The central result is in line with intuition: more coordination enhances efficiency. In this respect, particularly the sharing of information about the network and dispatch status between national TSOs can considerably lower redispatch volumes. Moreover, liquid intra-day markets, enabling cross-border 
counter-trading, and multilateral redispatch actions (MRAs), implemented in a flow-based congestion management setting, can levy further benefits. At the same time, redistributional effects between countries do exist and can be large. While results provide quantitative evidence for the study area, qualitative insights apply to other regions. Particularly, we picked our study region for two reasons: first, its electricity markets are already closely connected and further integration is envisaged. Second, the issue of loop flows from northern Germany to Bavaria and Austria, putting pressure on the Polish and Czech systems, is subject to controversial debate ${ }^{4}$. Therefore, it provides an interesting and politically relevant application.

The remainder of this paper is structured as follows. Section 2 presents the model formulation and describes the cases of coordination as well as the underlying data. Section 3 reports on the results and discusses their significance. Section 4 concludes and outlines avenues for future research.

\section{MODEL FORMULATION}

The general structure of our numerical model resembles the current European spot market design, consisting of a day-ahead market with uniform pricing and a subsequent curative congestion management phase. The day-ahead or spot market accounts for transmission limitations only on international crossborder trade. Internal physical network restrictions are considered outside the spot market in the congestion management approach by the responsible TSO (Kunz, 2012).

To this end, we employ two distinct models: the spot market is modeled as a cost minimization problem, where cross-border transmission lines are represented by a transportation model. The model accounts for both commitment and dispatch of individual generation units and is optimized for an entire year using a rolling planning approach. Key results are the schedules of generation units and international exchanges. Based on these data, the congestion management is carried out, reflecting different cases of international coordination. Importantly, both stages of the model are not connected by any kind of feed-

\footnotetext{
${ }^{4}$ See e.g. ČEPS et al (2013) and THEMA (2013).
} 
back mechanism. In the following, we reduce our model description to the most relevant aspects. A detailed account of the spot market model can be found in Abrell and Kunz (2015).

\subsection{The Spot Market Model}

The spot market model minimizes total system cost (1) consisting of generation and startup cost. The market clearing constraint (2) ensures the zonal balance of residual load, conventional generation, pumped-hydro generation and pumping, as well as international exchanges. Conventional generation levels are restricted by minimum and maximum capacities in case the unit is operating (3). Pumped-hydro storages are characterized by their maximum generation, pumping, and storage capacities. The spot market model abstracts from congestion within countries, whereas exchanges between different market zones (countries) are restricted by the available commercial trading capacity (4), the net transfer capacity (NTC).

$$
\begin{array}{cc}
\min _{G_{p, t}, U P_{p, t}} \sum_{p, t}\left(m c_{p} G_{p, t}+s c_{p} U P_{p, t}\right) & \\
\sum_{n \in c}\left(q_{n, t}-g_{n, t}^{R E S}\right)=\sum_{p \in c} G_{p, t}+\sum_{s \in c}\left(V_{s, t}-W_{s, t}\right)+\sum_{c c}\left(T F_{c c, c, t}-T F_{c, c c, t}\right) & \forall c, t \\
O N_{p, t} g_{p}^{\text {min }} \leq G_{p, t} \leq O N_{p, t} g_{p}^{\max } & \forall p, t \\
T F_{c, c c, t} \leq n t c_{c, c c} & \forall c, c c, t \\
T F_{c, c c, t}, G_{p, t}, V_{s, t}, W_{s, t} \geq 0 & \forall c, c c, p, s, t \\
O N_{p, t}, U P_{p, t} \in\{0,1\} & \forall p, t
\end{array}
$$

\subsection{The Congestion Management Model}

The congestion management model determines the redispatch of generation units given the received spot market schedules. In case physical network capacities do not suffice to accommodate the pattern of electrical flows, redispatch is carried out. Redispatch describes the increase or decrease of spot market generation and is performed in a cost-minimizing fashion considering the physical characteristics of the transmission network. Thus, a plant that increases generation is compensated by the transmission 
system operator for additional costs, whereas a plant that decreases generation must refund its saved generation cost. The physical load flow is calculated using a DC load flow approach, where we abstract from transmission losses (Leuthold et al., 2012). The general formulation of the model is given below - a detailed description of the congestion management issue and the model formulation can be found in Kunz (2012) and Abrell and Kunz (2015).

$$
\begin{array}{cc}
\min _{G_{p, t}^{C M}, C U R_{n, t}^{r e s}, C U R_{n, t}^{\text {load }}} \sum_{p, t} m c_{p} G_{p, t}^{C M}+\sum_{n, t}\left(c^{C, r e s} C U R_{n, t}^{\text {res }}+c^{C, \text { load }} C U R_{n, t}^{\text {load }}\right) & \\
q_{n, t}-g_{n, t}^{R E S}=\sum_{p \in n}\left(g_{p, t}^{D A}+G_{p, t}^{C M}\right)+\sum_{s \in n}\left(v_{s, t}^{D A}-w_{s, t}^{D A}\right)-C U R_{n, t}^{r e s}+C U R_{n, t}^{\text {load }}+N I_{n, t} & \forall n, t \\
o n_{p, t}^{D A} g_{p}^{\text {min }} \leq g_{p, t}^{D A}+G_{p}^{C M} \leq o n_{p, t}^{D A} g_{p}^{\max } & \forall p, t \\
\left|\sum_{n} p t d f_{l, n} N I_{n, t}\right| \leq p_{l}^{\max } & \forall l, t \\
C U R_{n, t}^{r e s} \leq g_{n, t}^{R E S}, C U R_{n, t}^{\text {load }} \leq q_{n, t}, G_{p, t}^{C M}, N I_{n, t} \text { free } & \forall n, t
\end{array}
$$

Similar to the spot market model, costs are minimized, defined as the sum of positive and negative generation redispatch, valued with marginal generation cost (5). Equation (6) formulates the nodal energy balance, which determines the net input into the physical transmission network and ensures balance of supply and load at nodal level. Generation units are limited in their redispatch contribution by the respective minimum and maximum capacities and the commitment in the spot market (7). Moreover, curtailment of renewables and load is possible in case redispatch capacities do not suffice to ensure feasible network flows. Individual transmission lines are characterized by their maximum capacity, which sets bounds on the flows determined by the power transfer distribution factor (PTDF) matrix (8). This model specification, (5-8), assumes perfect coordination of congestion management in the entire system as it abstracts from country-specific restrictions on cross-border redispatch. In the following, we introduce different cases of coordination by imposing additional constraints. 


\subsection{Cases of Coordinating Congestion Management}

Electrical flows depend on the technical properties of the entire transmission network. Thus, a certain fraction of a transaction flows on the direct link between both transaction points, whereas the remaining fraction follows alternative routes. Nodal in-feeds and withdrawals, thus, have widespread implications on transmission flows in highly meshed electricity systems. While continental Europe is linked by a synchronous transmission network, the system is characterized by a patchwork of different, mostly national, TSOs, electricity market specifications, and national jurisdictions. A perfectly coordinated approach to congestion management thus would require, among others, an intensive sharing of information and common network management approaches.

We define four cases of cross-country coordination, which differ in the degree of information sharing, the access to cross-country redispatch capacities, the geographical balancing areas, and the mode of cross-country allocation of network capacities for redispatch. Specifically, we analyze two limiting cases: no coordination (Case 1) and perfect coordination including multilateral redispatch actions (Case 4). In between, we take information sharing, for which actual vehicles exist by $2015^{5}$, into account (Case 2), as well as possibilities for cross-border counter-trading (Case 3).

\section{Case 1: Uncoordinated Congestion Management without Information Sharing}

This first case has a purely national focus. Any cross-border sharing of information on transmission flows or responsibility is absent. To this end, we introduce equation (9) which imposes a national balance of redispatch actions and disallows contribution of redispatch options from neighboring countries.

$$
\sum_{p \in c} G_{p, t}^{C M}=0 \quad \forall c, t
$$

Moreover, we restrict the TSOs' responsibility to their own control area. We reformulate the optimization problem to a Generalized-Nash-Equilibrium (GNE) with different exogenous valuations of

\footnotetext{
${ }^{5}$ The agencies CORESO (see www.coreso.eu, accessed August 25, 2015), TSO Security Cooperation (TSC, see www.tscnet.eu, accessed August 25, 2015), or the Joint Allocation Office (JAO, see www.central-ao.com, accessed August 25, 2015) provide services of information sharing between European TSOs.
} 
transmission capacity constraints. We follow the approach developed by Oggioni et al. (2012) and apply the formulation described in Kunz and Zerrahn (2015). To this end, a separate objective function, parallel to (5), and separate constraints (6-8), are set up for each national $\mathrm{TSO}^{6}$, and access to plants in a country is only allowed for the respective TSO. At the same time, national TSOs target their redispatch actions solely to alleviate congestion in their own control area and directly connected cross-border lines. Information on neighboring electricity systems remains hidden to them. In that way, coordination is restricted.

\section{Case 2: Coordinated Congestion Management with Information Sharing}

The second case introduces coordination by revealing information on neighboring electricity systems. In this way, the effects of its own actions on adjacent systems are known for each TSO, or some entity coordinating its actions. Formally, the valuation of all network constraints is now identical across all countries, and the complete network information as well as its equal valuation is taken into account in the minimization. At the same time, each region has to keep up its energy balance, as imposed by equation (9). The mathematical formulation comprises equations (5-9), as given above, where the single objective function for one supra-national entity (5) can be regarded as equivalent to the coordination case between TSOs described above.

\section{Case 3: Coordinated Congestion Management with Counter-Trading}

Beyond information sharing, the third case introduces access to redispatch capacities in neighboring countries through counter-trading. In this respect, counter-trading means that redispatch capacities can be exchanged across countries, for example in intraday markets, to counterbalance own redispatch actions. This is formally achieved by abandoning the national energy balance (9) and instead introducing an augmented version (10) to the initial problem (5-8). Cross-border market transactions are upper bounded by the available net transfer capacity and the corresponding spot market schedules as required by equation (11).

\footnotetext{
${ }^{6}$ For convenience, we assume one TSO for Germany, although, in fact, there are four. The benefits of coordination between these four German TSOs are analyzed in Kunz and Zerrahn (2015).
} 


$$
\begin{array}{cr}
\sum_{p \in c} G_{p, t}^{C M}=\sum_{c c}\left(T F_{c, c c, t}^{C M}-T F_{c c, c, t}^{C M}\right) & \forall c, t \\
0 \leq t f_{c, c c, t}^{D A}+T F_{c, c c, t}^{C M} \leq n t c_{c, c c} & \forall c, c c, t \\
T F_{c, c c, t}^{C M} \text { free } & \forall c, c c, t
\end{array}
$$

\section{Case 4: Coordinated Congestion Management with Multilateral Redispatch Actions}

The fourth case represents perfect coordination among national TSOs and extends the access to neighboring redispatch capacities through the introduction of multilateral redispatch actions. While in the previous case cross-country redispatch is restricted to counter-trading, limited by market-related NTCs, multilateral redispatch actions, which are typically performed outside the market, are limited by the physical transmission capacity. This case therefore reflects either a single TSOs optimizing the entire transmission network, or equivalently a set of national TSOs with perfect information sharing and common network management procedures. As actual physical cross-border network capacities, instead of calculatory NTCs, are applied, this case can also be described as flow-based congestion management. To restrict re-optimization beyond congestion management, we allow redispatch only in hours in which some link in the network is subject to congestion, and add equation (12), disallowing positive profits. Formally, equations (5-8) and (12) characterize the mathematical problem.

$$
\sum_{p, t} m c_{p} G_{p, t}^{C M}+\sum_{n, t}\left(c^{C, \text { res }} C U R_{n, t}^{r e s}+c^{C, \text { load }} C U R_{n, t}^{\text {load }}\right) \geq 0 \quad \forall t
$$

\subsection{Data}

Our application focuses on congestion management in the central eastern European region, covering Germany, Poland, Czech Republic, Slovakia, and Austria. These countries are considered on a detailed level regarding generation units and transmission infrastructure. To account for international exchanges and flows, the remaining European countries are modeled on nationally aggregated levels. A 
detailed description of the underlying data is given in Egerer et al. (2014). In contrast to this, our dataset is calibrated to the year 2013; further adjustments are described in the following.

Generation is differentiated between non-dispatchable renewable and dispatchable conventional technologies. For solar PV and wind power, we apply hourly feed-in time series for 2013. For Germany, the time series are derived from national TSOs (50Hertz, 2015; Amprion, 2015; TenneT, 2015; TransnetBW, 2015). For all other countries, re-analysis weather data is used (ECMWF, 2015). Renewable generation enters the model at zero marginal costs, but can be curtailed at cost of 75 Euro/MWh. For conventional generation, we differentiate between generation technologies and fuels, which are characterized by different technical specifications such as efficiency, minimum generation and operating times. The assumptions on minimum generation and operating times follow Abrell and Kunz (2015). Marginal generation costs of conventional units are based on fuel and $\mathrm{CO}_{2}$ certificate prices for 2013 . Electrical load is considered as a fixed and price-inelastic hourly demand. Profiles are extracted from ENTSO-E (2015a).

The transmission network includes the high-voltage level of 220 and $380 \mathrm{kV}$ and comprises 1397 transmission circuits and 681 substations. To capture interactions with electricity systems in neighboring countries, an aggregated PDTF matrix is determined. We first calculate the PTDF matrix for the entire ENTSO-E network and then aggregate the network in neighboring European countries. To this end, neighboring countries are connected to the detailed network of the investigated region through an equivalent network with lower level of detail. This approach allows to approximate the impact of neighboring electricity systems on load flows in the detailed network. In contrast, the spot market model relies on a transportation model of the transmission network, which reflects commercial transactions rather than physical load flows. Commercial transactions are bounded by NTCs, for which we employ hourly day-ahead values for 2013 for the study region, based on CAO (2015). For other European countries, net transfer capacities are considered on a yearly basis (ENTSO-E, 2015b). 


\section{RESULTS AND DISCUSSION}

As described in Section 2, our modelling approach comprises two steps. First, spot market dispatch and unit commitment based on transactional trade-flows between market zones, where zones reflect countries, except for the single German-Austrian market area. Given the spot market commitments, congestion management measures are evaluated in a second step with differing levels of coordination between market zones. It is important to note that the spot market commitments are identical throughout the different congestion management cases to ensure comparability of the congestion management cases. In the following, we will first describe the spot market results and subsequently present and discuss the results of the congestion management cases.

\subsection{Spot market dispatch and congestion patterns}

The spot market generation pattern basically resembles the European electricity market in 2013 . Table 1 shows the simulated generation volumes of the study region. Results for the remaining European countries indicate that Italy sees the highest net imports, followed by the Netherlands, and Belgium. Main exporters of electricity are France, followed by Germany, and Czech Republic. This typical pattern adequately reproduces the historical market outcome in 2013. Countries with cheap generation capacities like renewables, nuclear, or lignite, replace rather expensive gas- and oil-fired generation capacities in countries like Italy, the Netherlands, and Belgium in the course of of significant international exchanges.

Renewable generation is a dominating source in Germany and contributes approximately $23 \%$ to the total generation. In combination with nuclear, lignite, and coal generation, this results in significant net exports in the range of $29 \mathrm{TWh}$. In other countries, renewable generation shares are substantially lower and conventional technologies dominate national generation portfolios. The share of renewable generation is one relevant aspect, as it implies a shift in the spatial generation pattern and thus the use of the transmission network. 
Table 1. Results of the spot market model: generation and consumption in TWh

\begin{tabular}{lccccc}
\hline & AT & CZ & DE & PL & SK \\
\hline Total Generation, of which & $\mathbf{6 3 . 3}$ & $\mathbf{8 7 . 7}$ & $\mathbf{5 8 3 . 1}$ & $\mathbf{1 4 9 . 7}$ & $\mathbf{2 8 . 3}$ \\
\hline Hydro & 36.9 & 2.2 & 10.1 & 1.3 & 3.9 \\
\hline Nuclear & - & 26.6 & 92.1 & - & 15.0 \\
\hline Lignite & - & 48.6 & 150.1 & 64.1 & 3.8 \\
\hline Coal & 7.3 & 5.4 & 129.6 & 73.2 & 1.3 \\
\hline Gas & 7.4 & 0.5 & 51.1 & 1.4 & 2.2 \\
\hline Oil & 2.6 & 0.1 & 3.8 & 2.1 & 0.1 \\
\hline RES (Wind, Solar, and Biomass) & 6.1 & 2.7 & 137.5 & 6.4 & 0.8 \\
\hline PSP & 3.0 & 1.7 & 8.4 & 1.0 & 1.3 \\
\hline Consumption & $\mathbf{6 9 . 6}$ & $\mathbf{6 2 . 8}$ & $\mathbf{5 4 3 . 6}$ & $\mathbf{1 4 5 . 5}$ & $\mathbf{2 6 . 7}$ \\
\hline
\end{tabular}

The spatial generation pattern has two implications for flows in the European transmission network. First, the transmission network is often insufficient to transport that energy, which results in an increasing number of congestion situations. Congestion management measures are thus necessary to reduce individual line overloading to ensure secure network operation. Second, loop flows in adjacent electricity systems extend this need also to neighboring countries as additional congestion issues and management needs can arise.

Figure 2 depicts the congestion event hours for internal and cross-border transmission lines. As an indicator for the congestion frequency, they are defined as the sum of hourly overloaded transmission elements. The main fraction of congestion events (4894 h) occurs within Germany. Congestion events in other countries are significantly lower, with the highest number of events in Poland (1043 h). Cross-border transmission lines are restrictive in 1839 cases, where all events occur on German cross-border links to neighboring countries. Thus, nearly $85 \%$ of all congestion events occur either in Germany or on connecting cross-border links. 
Figure 2. Internal and cross-border congestion

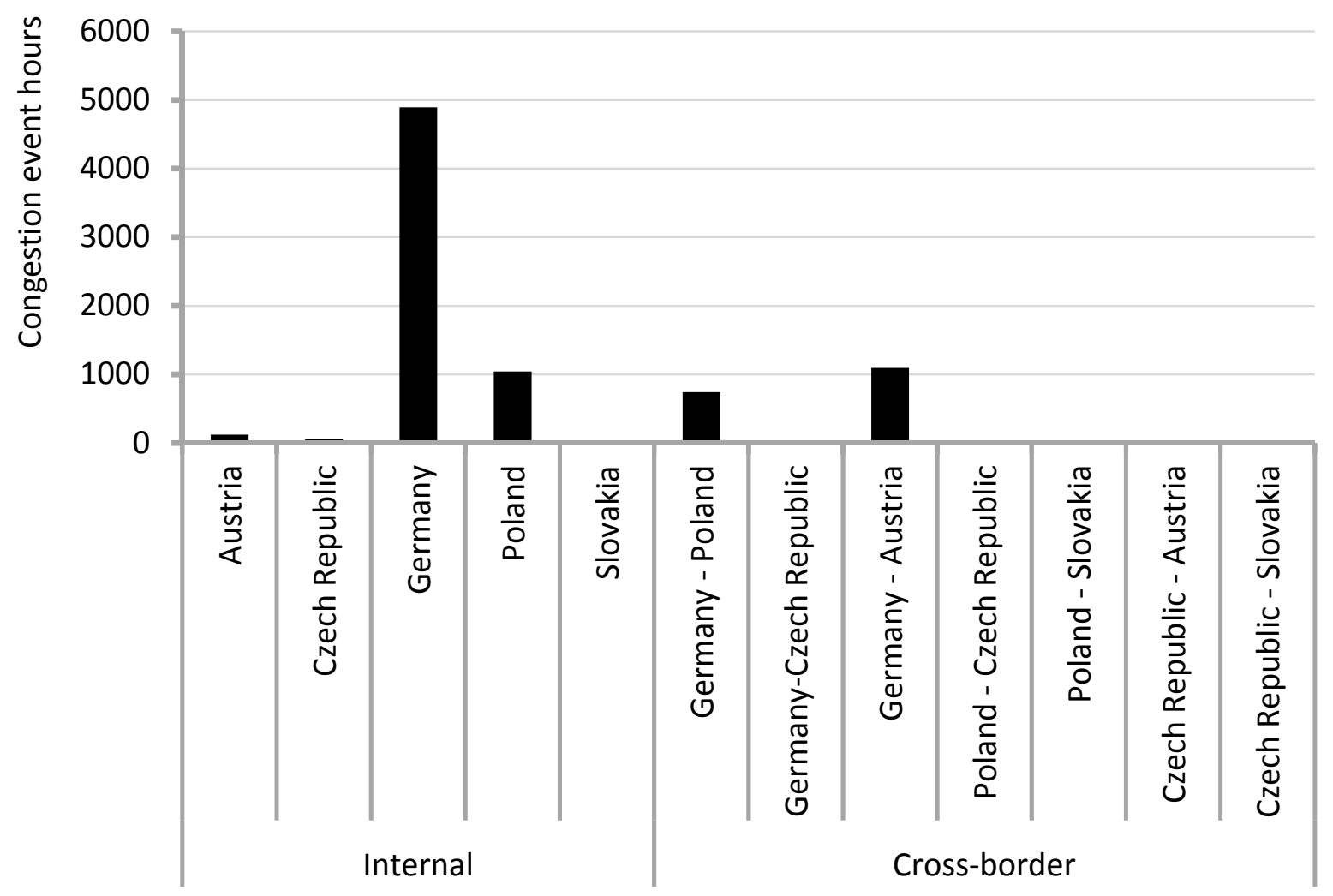

The hourly realization of commercial and physical cross-border flows between Germany and Poland, as well as between Poland and Czech Republic, is illustrated in Figure 3, and Figure 4, respectively. Due to the network characteristics and significant energy exports from Germany, a certain fraction of the generated energy will be transported through the meshed transmission network via Poland and Czech Republic to southern Germany. This energy is not reflected in the commercial spot market trade schedule, which furthermore sees flows in the opposite direction, in particular at the German-Polish border. Results thus confirm the effect that physical cross-border flows are higher than commercial transfers, and thus to some extent decoupled. This highlights that national generation patterns entail implications on adjacent system through the meshed transmission network. 
Figure 3. Hourly physical and commercial flows from Germany to Poland in MW

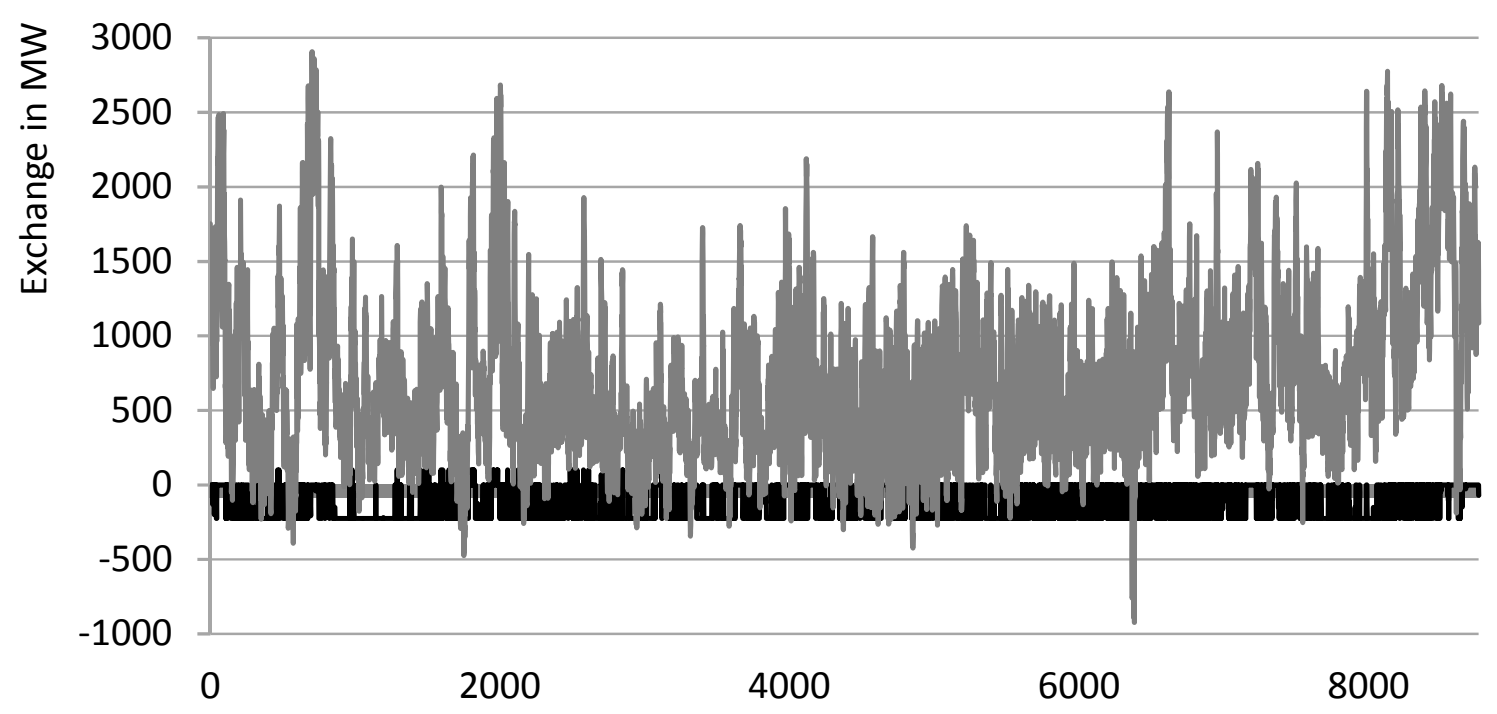

Hour

—Commercial Transfer DE > PL_—Physical Transfer DE > PL

Figure 4. Hourly physical and commercial flows from Poland to Czech Republic in MW

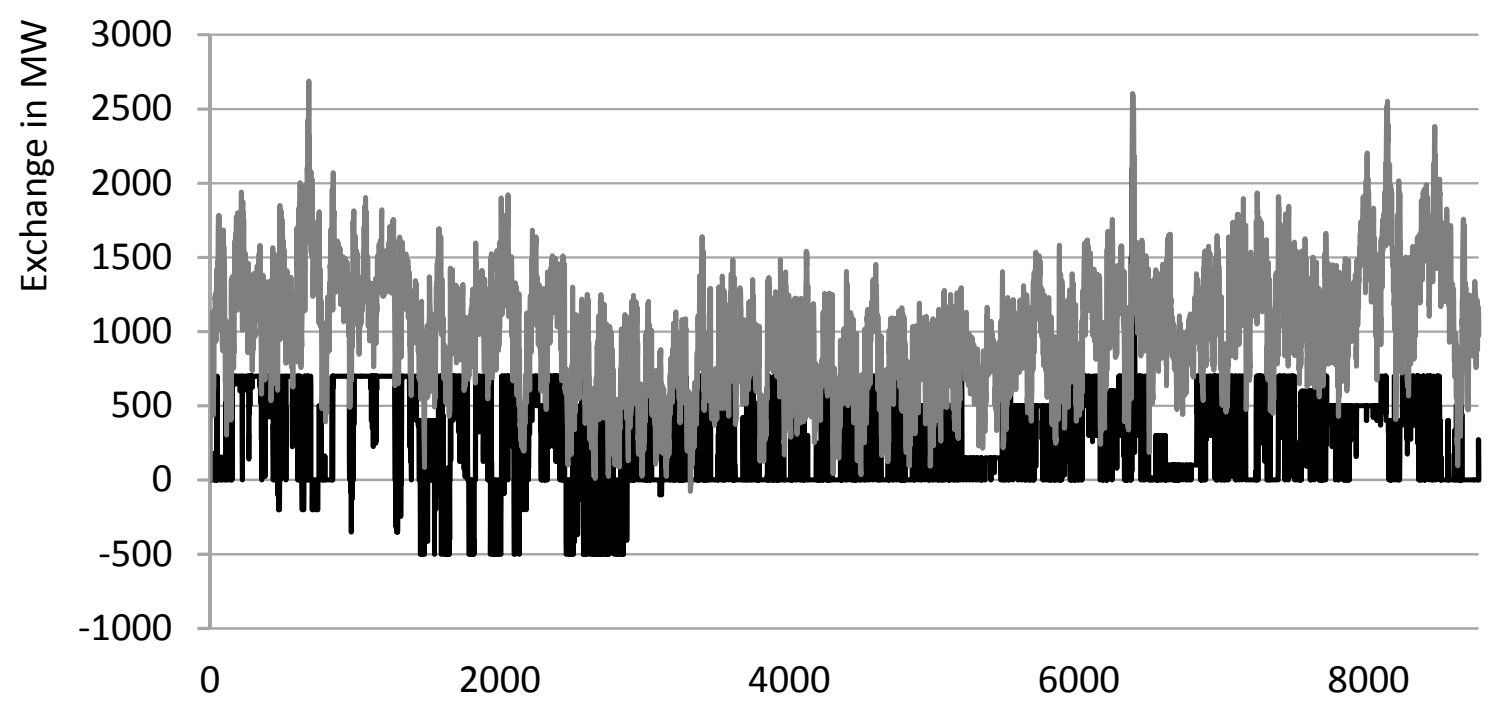

Hour

—Commercial Transfer PL > CZ — Physical Transfer PL > CZ 


\subsection{Redispatch}

Given the inter-dependencies arising from the international transmission flow pattern, we now analyze the management of network congestion under different levels of coordination between TSOs. Figure 5 depicts the total costs of the four different coordination cases, as described in Section 2. The central result is in line with intuition: total costs for congestion management strongly decrease for higher levels of coordination. While for the uncoordinated GNE case (Case 1) costs are above 350 million Euro, they gradually decrease with higher degrees of coordination to 70 million Euro for optimized congestion management with MRAs and flow-based cross-border capacity allocation (Case 4). The intermediate Cases 2 and 3 entail costs of 138 million Euro and 81 million Euro, respectively. We also calculated the limiting case of a nodal pricing framework. Integration of network constraints into spot market dispatch and unit commitment yields total cost savings of 102 million Euro, compared to the NTC-based spot market dispatch with national copperplate assumptions. As this paper focuses on the beneficial impacts of improving coordination in a separated congestion management phase, we do not pursue this thread further.

\section{Figure 5. Total Congestion management cost in million Euro, broken down into components}

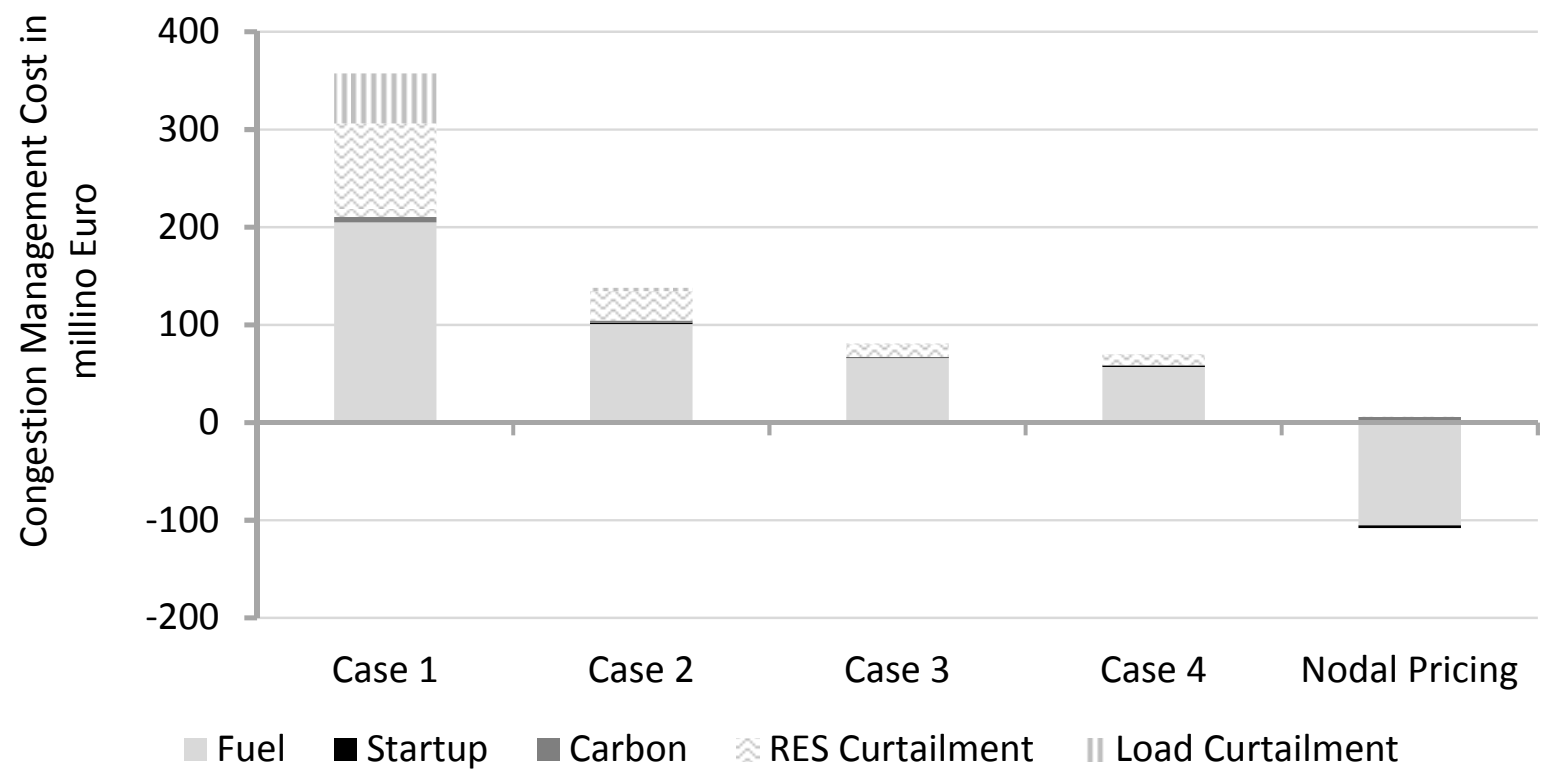

The redispatching of plants accounts for the biggest share among congestion management costs. Particularly, the bulk of the savings accrues from reduced expenses for fuel. Start-up cost only play a 
minor role. The basic rationale is straightforward: more coordination enables access to cheaper and more effective redispatching capacities and thus increases efficiency of congestion management. The absolute amount of renewables curtailment, accounting for around $15-25 \%$ of congestion management costs in all cases, is reduced as well. Load curtailment is interpreted as infeasibility measure in case the model does not deliver a solution by drawing on the standard options. Its costs of 5,000 Euro per MW reflect the value of lost load. As such, it is only of relevance in the uncoordinated GNE case (Case 1), in all other scenarios, it adds less than $0.5 \%$ to total costs, in the coordinated case with information sharing (Case 2) $2.5 \%$. As it is unlikely that, in an ever more integrated electricity system, no coordination at all is implemented, we regard Case 1 as a theoretical limiting case. However, the high level of unmatched load and curtailment of renewables highlights the importance of coordination in network operation to increase security of supply in the European transmission system.

Table 2. Total congestion management costs in million Euro, redispatch volumes in TWh, and specific redispatch costs in Euro/MWh

\begin{tabular}{lcccc}
\hline Case & Total Cost & $\begin{array}{c}\text { Negative } \\
\text { Redispatch } \\
\text { Volume }\end{array}$ & $\begin{array}{c}\text { Total } \\
\text { Redispatch } \\
\text { Volume }\end{array}$ & Specific \\
& & 5.6 & 11.2 & 55.4 \\
\hline Case 1: Uncoordinated CM & 357.5 & 3.1 & 6.2 & 43.1 \\
\hline Case 2: Coordinated CM with & 137.8 & & & \\
information sharing & & 2.8 & 5.5 & 29.1 \\
\hline Case 3: Coordinated CM with & 80.8 & & & \\
counter-trading & & 3.8 & 7.5 & 18.6 \\
\hline Case 4: Coordinated CM with & 70.0 & & & \\
MRAs & & & & \\
\hline
\end{tabular}

The effect of coordination on the total redispatch volume is not monotone, see Table 2 . The total negative redispatch volume for the uncoordinated GNE case (Case 1) amounts to about 5.6 TWh. Moving 
to an integrated optimization with information sharing, Case 2, effects of generation changes are taken into account for the entire network, and redispatch volumes accordingly decrease to $3.1 \mathrm{TWh}$. Widening the energy balancing area by allowing for cross-border counter-trading yields a lower redispatch volume of 2.8 TWh in Case 3. Allowing for MRAs in a flow-based redispatch setup, Case 4, enables a greater set of feasible actions, and increases the volume to $3.8 \mathrm{TWh}$.

Combining the two patterns to calculate the specific costs indicates efficiency gains in a common metric. Specific costs are defined as costs for redispatch and renewable curtailment divided by the negative redispatch volume, thus disregarding load curtailment. ${ }^{7}$ They can be interpreted as the average costs for relieving a unit of overload on a transmission link. The right column of Table 2 gives an overview of the specific costs in Euro per MWh. Inspection highlights two channels through which efficiency gains from coordination arise: a volume and specific cost-effect. We discuss below how both effects relate to the different steps toward more coordination.

\section{Common Responsibility and Information Sharing - Cases 1 and 2}

The volume effect is isolated when moving from the uncoordinated GNE Case 1, with five independent TSOs responsible for their own control area, to Case 2, with appropriate information sharing of congestion for the entire region. The latter case is equivalent to a single TSO operating the network in the entire region, however subject to zonal balance restrictions. While specific costs decrease moderately, from 55 to 43 Euro per MWh, total costs drop by more than half to 138 million Euro. Savings thus arise mostly from the utilization of a lower number of units, where the negative redispatch volume decreases by

\subsection{TWh.}

The channel through which lower redispatch volumes and costs arise is the sharing of information. When moving from Case 1 to 2, five TSOs are replaced by one entity with information for the entire study region. Congestion in one country can thus be removed by drawing on resources in another country. At the same time, the information sharing between regions prevents that redispatch in one country may create new congestion in the system of an adjacent region. Access to these substantial efficiency gains requires a

\footnotetext{
${ }^{7}$ Qualitative results including load curtailment are analogous.
} 
common information infrastructure on operational network data across borders. Each national TSO should have the information on the congestion situation, availability of resources and network topology in all countries. In this way, effects of its own orders on adjacent systems could be assessed, and those remedial actions in the network could be identified which relieve congestion in a more efficient way, wherever they are best undertaken. In this regard, it is irrelevant whether multiple TSOs coordinate on optimal measures, or one superordinate entity provides a redispatch plan for the entire region, as modeled in our Case 2.

In the European context, operational data is commonly exchanged among TSOs through, for example, daily day-ahead and hourly intraday congestion forecasts by TSO co-operations like TSC and CORESO. Thus, TSOs are regularly informed about network restrictions on a European scale and can incorporate them into their operational planning. As information sharing does take place to a certain extent, the uncoordinated Case 1 can therefore be seen as a limiting scenario to shed light on the gains of a common information pool from an academic perspective.

\section{Proposition I}

Cross-border coordination in congestion management by sharing information for the entire region substantially reduces costs, as resources in all countries can be used to remove overflows in any country. Moreover, information sharing prevents redispatch which induces new network congestion beyond a coordinated optimum. Efficiency gains mainly arise through lower redispatch volumes.

\section{Counter-trading and Cross-border Balancing - Cases 2 and 3}

Case 3 introduces cross-border counter-trading. National TSOs can order generation increases in an adjacent country to set off decreases in their own country. All cross-border actions are limited by the available NTC after spot market clearing. As a direct effect, the total volume of congestion management measures decreases, from 3.1 TWh to $2.8 \mathrm{TWh}$, as alterations within one country do not have to be balanced within the country, but cross-border redispatch can set each other off. The slight decrease in total volume is accompanied by a substantial drop in total costs, from 138 to 81 million Euro. Specific costs thus fall from 43 to 29 Euro per MWh, attributed to both less redispatch and renewables curtailment. At the same time, cross-border counter-trading has implications on the regional distribution of redispatch 
measures across countries. Figure 6 visualizes the absolute volumes of positive and negative redispatch by country. While these are roughly equal within each country under Cases 1 and 2, except for small deviations due to load curtailment, they deviate greatly in Case 3.

Figure 6. Positive and negative redispatch volumes by country across the four coordination cases in TWh

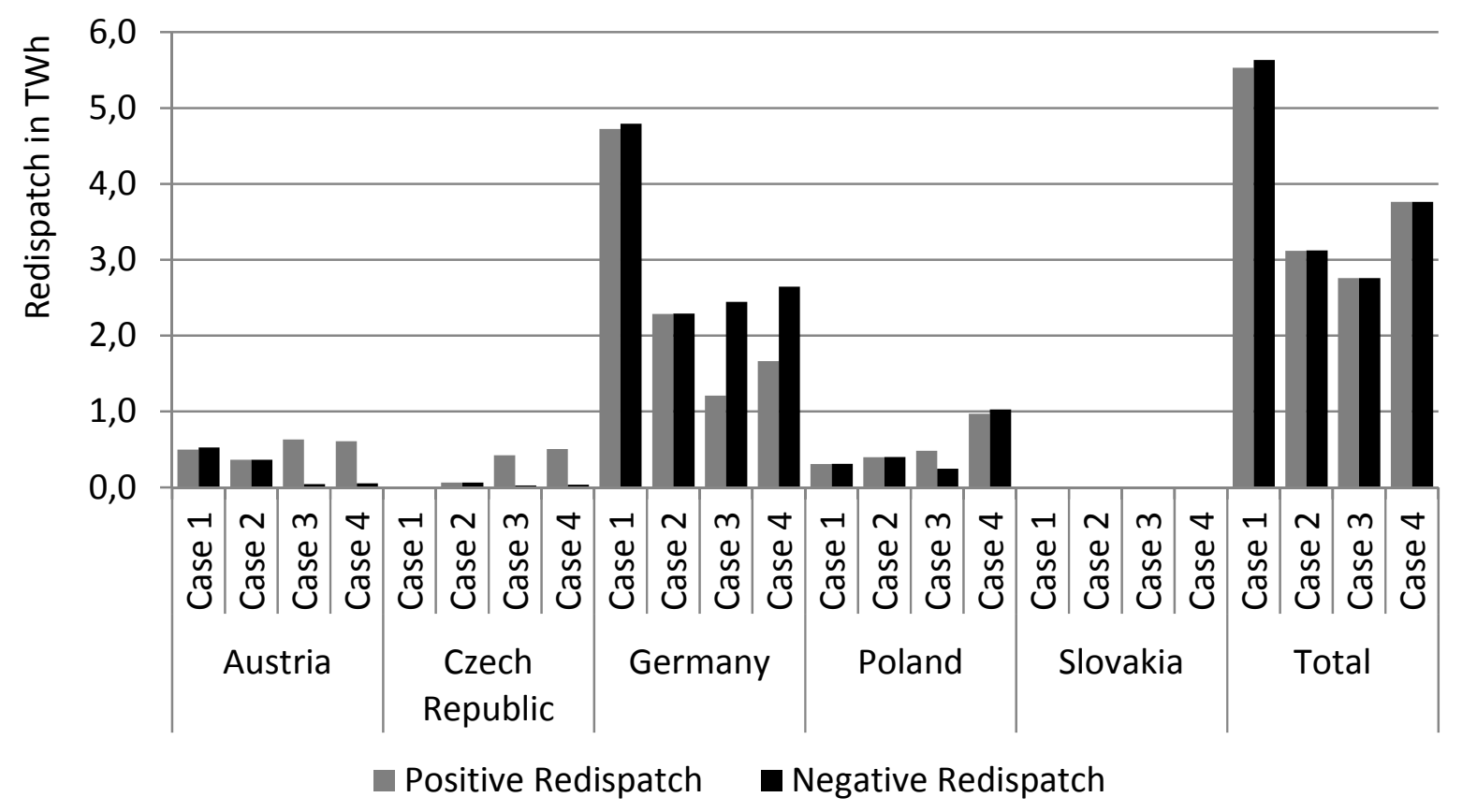

Without cross-border counter-trading, alterations of the generation dispatch have to be set off within each country, irrespective whether less costly alternatives in other countries exist. Allowing for counter-trading in Case 3, the energy balance can be fulfilled by drawing on cheaper units in other countries. Germany, for example, accounts for $88 \%$ of all generation decreases, but only $45 \%$ of all increases. Instead, redispatching resources within Austria, where $23 \%$ of all generation increases take place, but only $1 \%$ of all decreases, and the Czech Republic, with $15 \%$ of all increases and $1 \%$ of all decreases, are employed. Specific costs are thus lowered by directing negative redispatch toward rather expensive German plants, and positive redispatch towards cheaper plants in the other countries.

These efficiency gains can be levied by well-functioning cross-border intraday markets, which enable national TSOs or a supra-national entity to access sufficient resources. This allows TSOs to 
balance redispatch actions beyond national borders and increases the efficiency of congestion management actions. The effectiveness of counter-trading is, among others, affected by the liquidity of the intraday market, which might limit the potential to balance redispatch actions. As a caveat, our model abstracts from an anonymous intraday trading and therefore tends to overestimate the. In fact, TSOs might not know the effectiveness of counter-trading measures when contracting them through an anonymous intraday market. Nevertheless, the results highlight the beneficial potential of balancing redispatch actions on an international level.

\section{Proposition II}

An enlargement of the balancing area for congestion management can yield a substantial decrease in specific redispatch costs. It prevents costly energy balancing measures within each country, and instead opens access to the cheapest alternatives. Liquid intraday markets and administrative adjustments are the prerequisites.

Flow-based Cross-border Redispatch with - Cases 3 and 4

Case 4 abandons the NTC limitations for cross-border redispatch and instead determines the scope of cross-country congestion management based on physically available capacities. We term this degree of flexibility flow-based congestion management, as an underlying grid model captures the physically feasible cross-border electricity flows. Intuitively, the total redispatch volume increases, from 2.8 to 3.8 TWh, while total costs fall from 81 to 70 million Euro. The greater flexibility in exploiting cross-border network capacities enables access to further cheaper units for generation upshifts which replace the upshift of more expensive national units. Accordingly, specific costs drop from 29 to 19 Euro per MWh.

When interpreting results, a caveat applies: in Case 4, costs for congestion management can be interpreted as lower bound. This is due to the model setup allowing a certain degree of re-optimization: consider a case in which some line within a country is congested and the capacity of cross-border links is not fully exploited. If the spot market dispatch is subject to NTC market coupling and commercial flows result in physical flows below the actual cross-border capacity limit, then the spot market does not necessarily deliver the least-cost dispatch subject to physical line capacities. In the redispatch phase, thus, 
generation adjustments may not only relieve overflows, but also achieve a lower cost market clearing expost. In Case 4, this effect can be present, but it is restricted by two constraints. First, in hours without any congestion, redispatch is prohibited. Second, if redispatch occurs, then total costs may not be lower than under the spot market dispatch, implemented by the zero profit condition equation (12). At most, positive redispatch costs may be countervailed.

It is, however, difficult to disentangle to which extent re-optimization drives coordination gains. To give some assessment, we relax both constraints and calculate the limiting case exploiting the full reoptimization potential. As a result, total redispatch costs decrease to 43.8 million Euro. The additional gains of approximately 25.9 million Euro occur on a basis of approximately 5,900 hours, corresponding to 4,400 Euro of savings in an average hour of pure re-optimization. Total gains from multilateral redispatch of 10.7 million Euro, comparing Cases 3 and 4, arise in 1,900 hours. This corresponds to 5,700 Euro of savings in an average hour of multilateral and flow-based redispatch. This difference gives some indication that the cost decrease, when moving from Case 3 to 4 , is to a non-negligible extent driven by greater flexibility for redispatch measures and not only re-optimization incentives.

These efficiency gains can be accessed through an integrated congestion management approach for the entire region, where TSOs take into account multilateral redispatch actions and allocate crossborder network capacities according to the calculation of resulting physical flows using an accurate grid model. The establishment of common redispatch procedures, as envisaged in the CACM Network Code, may provide a suitable vehicle toward greater levels of coordination.

\section{Proposition III}

Flow-based allocation of cross-border network capacities for redispatch and coordinated multilateral redispatch actions substantially decrease total costs. The greater flexibility enables access to cheaper generation, which was kept out by the more restrictive NTC mechanism. In such setting, also reoptimization beyond targeting network congestion is possible. 


\subsection{Distribution of Congestion Management Costs}

The analysis of total costs highlights the overall gains from coordination. As multiple countries are involved, also the distributional perspective is of great relevance when it comes to incentives for further integration. Table 3 shows how total costs, excluding load curtailment, are distributed across countries. Here, costs for redispatch are assigned to that country where the corresponding generation unit is located. ${ }^{8}$

Table 3. Distribution of congestion management costs (excluding load curtailment) across countries in million Euro

\begin{tabular}{llllllll}
\hline Case & & AT & CZ & DE & PL & SK & Total \\
\hline Case 2: Coordinated CM & 17.5 & 3.7 & 103.2 & 10.1 & 0.0 & 134.7 \\
$\begin{array}{l}\text { with information sharing } \\
\text { Case 3: Coordinated CM }\end{array}$ & & & & & & \\
with counter-trading & 31.9 & 8.9 & 28.8 & 10.6 & 0.1 & 80.3 \\
\hline $\begin{array}{l}\text { Case 4: Coordinated CM } \\
\text { with MRAs }\end{array}$ & 27.9 & 9.0 & 29.4 & 3.5 & 0.0 & 69.9 \\
\hline
\end{tabular}

In Case 2, where national energy balances are maintained, Germany bears the bulk of redispatch costs, 103 out of 135 million Euro in total, while Austria and Poland pay for smaller shares of 18 and 10 million Euro, respectively, and the Czech Republic and Slovakia are only marginally affected. This picture is reversed when cross-border counter-trading is introduced. Germany's burden is over-proportionally reduced to almost a quarter, 29 million Euro, while especially costs paid within Austria rise to 32 million, although total costs decrease. An analogous pattern emerges for flow-based redispatch in Case 4 . The rationale is straightforward: the easier the access to cheaper generation, to relieve overflows or to reoptimize the dispatch, the more such resources are utilized and the according payments accrue in the respective countries.

\footnotetext{
${ }^{8}$ For convenience, we do not discuss the limiting Case 1 at here.
} 
Contrasting these figures with national congestion patterns sheds light on the question who pays for what. To this end, we calculate the total excess energy which cannot be transported through the network and attribute it to the country in which the respective line elements are located. If a cross-border line is subject to congestion, we conservatively share the volume equally between countries. In Figure 7, the first bar within each country section indicates the share of a country in total congestion of the region: $87 \%$ of all congested network elements are located within Germany, 9\% in Poland, and 4\% in Austria, where for both countries these are mainly cross-border lines to Germany. In Case 2, congestion volume shares, represented by the second bar within each country section, roughly correspond to the redispatch cost shares. By introducing cross-border counter-trading, however, a substantial part of congestion management costs is transferred to Austria and Czech Republic, see Cases 3 and 4. Results thus indicate that in a multilateral context, such as the European electricity system, the spatial structure of redispatch actions and corresponding costs do not necessarily coincide with congestion events. This effect becomes even more important with higher degrees of coordination as envisaged by European policy.

\section{Figure 7. Congestion shares and cost shares across countries and cases}

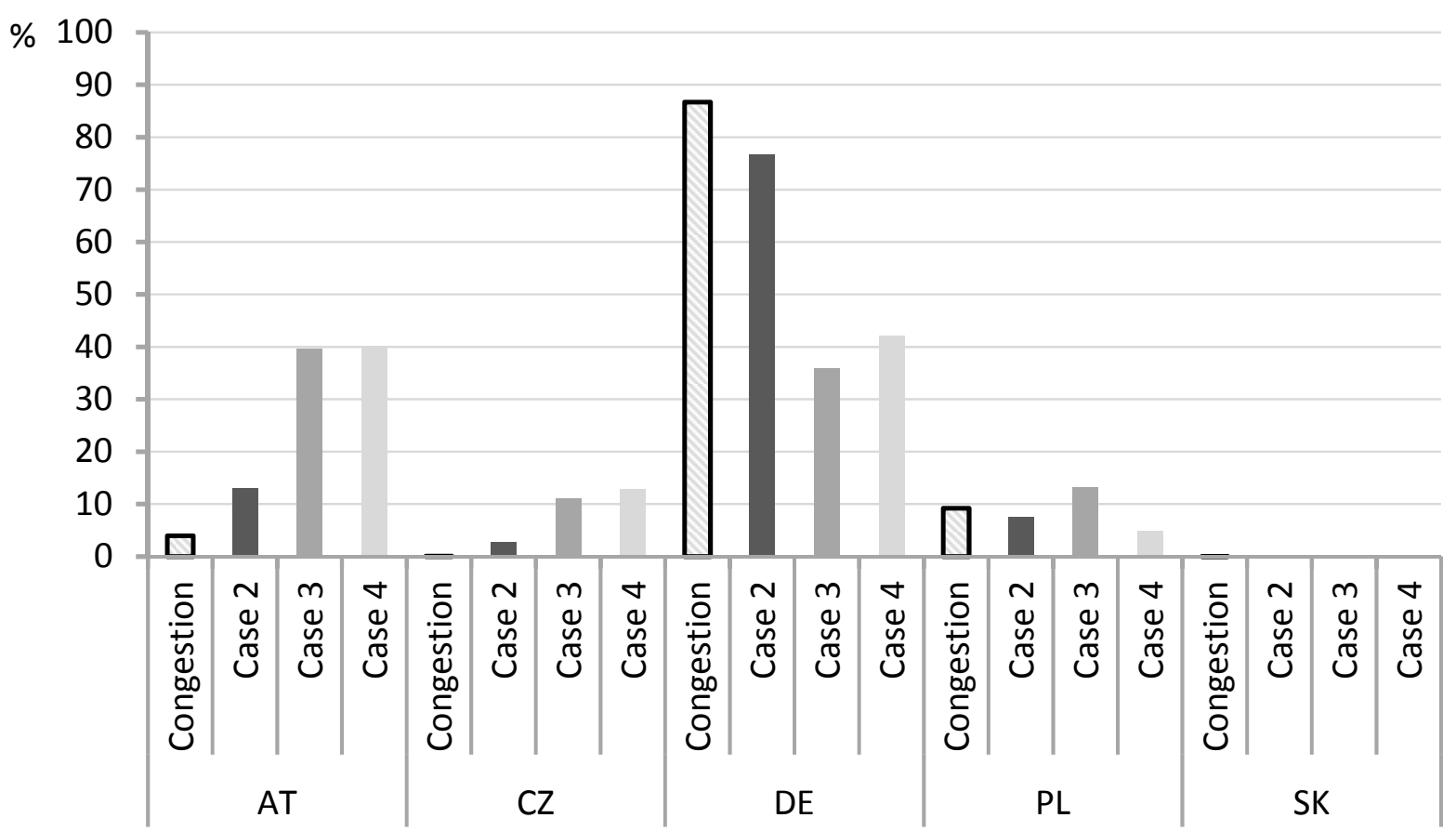

Note: bars indicate the share of each country in total congestion and cost volumes 
This uneven distribution of costs and benefits across countries may hamper incentives for closer cooperation. In the context of our results, Germany would have a strong incentive for closer cooperation in congestion management due to high coordination profits. On the other hand, Austria, Czech Republic, and Poland face higher costs in the coordinated cases than in the more national oriented Case 2 which may limit the willingness to coordinate on operational measures. What is thus needed to increase cooperation, is a mechanism to allocate congestion management costs in line with benefits on a national level. Among others, potential allocation mechanisms could follow the requesters-pay-principle or some network usage metric. However, how a particular cost allocation mechanism could be designed, is beyond the scope of this paper.

\section{Proposition IV}

Total cost savings are accompanied by considerable distributional effects. Particularly, countries in which more expensive generators are located gain from closer coordination while countries with cheaper generation lose. To align costs and benefits of coordinating cross-border congestion management, prudent mechanisms are required.

\section{CONCLUSIONS}

The reshaping of the European electricity generation structure poses new challenges on the electricity network. In the short run, congestion management through redispatch of power plants is a suitable means to maintain network stability. At the same time, European markets integrate ever closer. In this paper, we applied a large-scale model to the Central Eastern European region to shed light on the question whether and how the cross-border coordination of congestion management can be beneficial. The central result is in line with intuition: closer cooperation increases cost-efficiency by access to cheaper facilities. Particularly, the sharing of network information between national TSOs has the potential to considerably reduce redispatch volumes. Likewise, counter-trading, facilitated by liquid intra-day markets can decrease specific costs, and multilateral redispatch actions in a flow-based setting can levy further efficiency gains from coordination. At the same time, redistributional effects between countries can be large. Therefore, 
prudent cross-border cost allocation schemes are required to ensure incentive compatibility for all countries.

Several limitations and caveats remain. The questions how to sharply disentangle genuine coordination gains from re-optimization potential within the model mechanics, and how to implement modes of cooperation other than analyzed here, pose starting points for fruitful future research. Moreover, it remains open in this paper how market-based congestion management mechanism could provide additional benefits. Likewise, the question which allocation cost principles could reliably implement an incentive compatible mechanism for all parties remain to be investigated.

In this respect, the European Commission fosters a discussion about how to design closer cooperation between countries in the field of cross-border congestion management. While several coordination initiatives, like Coreso, TSC, or CAO (Capacity Allocation Office) exist, the CACM Network Code envisages the binding enactment of common rules and regulations. Our paper has shown that considerable welfare gains are indeed possible.

\section{ACKNOWLEDGEMENTS}

This work was carried out within the project MASMIE funded by the Mercator foundation. The authors thank Christian von Hirschhausen, Claudia Kemfert, Karsten Neuhoff as well as the participants of the Berlin Conference on Energy and Electricity Economics (BELEC 2015) for fruitful discussions and valuable comments. 


\section{REFERENCES}

50Hertz (2015). Grid Data. http://www.50hertz.com/en/Grid-Data

50Hertz, Amprion, TenneT, and TransnetBW (2015). EEG-Anlagenstammdaten. http://www.netztransparenz.de/de/Anlagenstammdaten.htm, accessed August 31, 2015.

Abrell, Jan, and Friedrich Kunz (2015). „Integrating Intermittent Renewable Wind Generation - A Stochastic Multi-Market Electricity Model for the European Electricity Market.” Networks and Spatial Economics 15: 117-147. dx.doi.org/10.1007/s11067-014-9272-4

Amprion (2015): Grid Data. http://amprion.de/en/grid-data, accessed August 31, 2015

Brunekreeft, Gert (2015). "Network unbundling and flawed coordination: Experience from the electricity sector." Utilities Policy 34: 11-18. dx.doi.org/ 10.1016/j.jup.2015.03.003

Bundesnetzagentur (BNetzA) (2012). Beschluss BK8-12-019. German Federal Network Agency (BNetzA). http://www.bundesnetzagentur.de/DE/Service-Funktionen/Beschlusskammern/1BKGeschaeftszeichen-Datenbank/BK8-GZ/2012/2012_0001bis0999/2012_001bis099/BK8-120019/BK8-12-019_Beschluss_BF.pdf?_blob=publicationFile\&v=2, accessed January 26, 2016. Bundesnetzagentur and Bundeskartellamt (BNetzA and BKartA) (2015). Monitoringbericht 2015. German Federal Network Agency (BNetzA), and German Federal Cartel Office (BKartA). http://www.bundesnetzagentur.de/SharedDocs/Downloads/DE/Allgemeines/Bundesnetzagentur/P ublikationen/Berichte/2015/Monitoringbericht_2015_BA.pdf?_blob=publicationFile\&v=3, accessed January 26, 2016.

Bundesnetzagentur and Bundeskartellamt (BNetzA and BKartA) (2014). Monitoringbericht 2014. German Federal Network Agency (BNetzA), and German Federal Cartel Office (BKartA). http://www.bundesnetzagentur.de/SharedDocs/Downloads/DE/Allgemeines/Bundesnetzagentur/P ublikationen/Berichte/2014/Monitoringbericht_2014_BF.pdf?_blob=publicationFile\&v=4, accessed January 26, 2016.

Bundesnetzagentur and Bundeskartellamt (BNetzA and BKartA) (2013). Monitoringreport 2012. German Federal Network Agency (BNetzA), and German Federal Cartel Office (BKartA). 
http://www.bundesnetzagentur.de/SharedDocs/Downloads/EN/BNetzA/PressSection/ReportsPubli cations/2012/MonitoringReport2012.pdf?_blob=publicationFile, accessed January 26, 2016.

CAO (2015). Daily Auction. Central Allocation Office (CAO). http://www.central-ao.com/capacityauctions/dialy-auction, accessed August 21, 2015.

Chaves-Ávila, José P., Reinier A.C. van der Veen, and Rudi A. Hakvoort (2014). "The interplay between imbalance pricing mechanisms and network congestion - Analysis of the German electricity market.” Utilities Policy 28: 52-61. dx.doi.org/ 10.1016/j.jup.2013.11.005

ČEPS, MAVIR, PSE, and SEPS (2013). Unplanned flows in the CEE region In relation to the common market area Germany - Austria. http://www.pse.pl/index.php?dzid=32\&did=1308, accessed August 31, 2015.

Egerer, Jonas, Clemens Gerbaulet, Richard Ihlenburg, Friedrich Kunz, Benjamin Reinhard, Christian von Hirschhausen, Alexander Weber, and Jens Weibezahn (2014). Electricity Sector Data for PolicyRelevant Modeling: Data Documentation and Applications to the German and European Electricity Markets. Data Documentation 72, German Institute for Economic Research. http://www.diw.de/documents/publikationen/73/diw_01.c.440963.de/diw_datadoc_2014-072.pdf, accessed August 31, 2015.

Egerer, Jonas, Jens Weibezahn, and Hauke Hermann (2015). „Two price zones for the German Electricity Market - Market Implications and Distributional Effects.” DIW Discussion Papers 1451. http://www.diw.de/documents/publikationen/73/diw_01.c.497246.de/dp1451.pdf, $\quad$ accessed August 31, 2015.

ENTSO-E (2015a). Hourly Load Values. European Network of Transmission System Operators for Electricity (ENTSO-E). https://www.entsoe.eu/db-query/consumption/mhlv-all-countries-for-aspecific-month, accessed August 31, 2015.

ENTSO-E (2015b). NTC Matrix. European Network of Transmission System Operators for Electricity (ENTSO-E). $\quad$ https://www.entsoe.eu/publications/market-reports/ntc-values/ntcmatrix/Pages/default.aspx, accessed January 26, 2016. 
ECMWF (2015). ERA Interim, Daily. European Centre for Medium-Range Weather Forecasts (ECMWF). http://apps.ecmwf.int/datasets/data/interim-full-daily/, accessed August 31, 2015.

European Commission (EC) (2015a). Energy Union Package. Communication COM(2015) 80 final.

European Commission (EC) (2015b). Commission Regulation (EU) 2015/1222 of 24 July 2015 establishing a guideline on capacity allocation and congestion management. Official Journal of the European Union.

European Commission (EC) (2009). Regulation (EC) No 714/2009 of the European Parliament and of the Council on conditions for access to the network for cross-border exchanges in electricity and repealing Regulation (EC) No 1228/2003. Official Journal of the European Union.

European Commission (EC) (2011). Energy Roadmap 2050. Communication COM(2011) 885 final.

Eurostat (2015). SHARES 2013 (Short Assessment of Renewable Energy Sources). http://ec.europa.eu/eurostat/web/energy/data/shares, accessed August 31, 2015.

Kunz, Friedrich (2012). "Improving Congestion Management - How to Facilitate the Integration of Renewable Generation in Germany." The Energy Journal 34(3): 55-78. dx.doi.org/ $10.5547 / 01956574.34 .4 .4$

Kunz, Friedrich, and Alexander Zerrahn (2015). "Benefits of Coordinating Congestion Management in Electricity Transmission Networks: Theory and Application to Germany." Utilities Policy 37: 3445. dx.doi.org/ 10.1016/j.jup.2015.09.009

Leuthold, Florian, Hannes Weigt, and Christian von Hirschhausen (2012). "A Large-Scale Spatial Optimization Model of the European Electricity Market.” Networks and Spatial Economics 12(1): 75-107. dx.doi.org 10.1007/s11067-010-9148-1

Neuhoff, Karsten, Julian Barquin, Janusz W. Bialek, Rodney Boyd, Chris J. Dent, Thilo Grau, Christian von Hirschhausen, Benjamin F. Hobbs, Friedrich Kunz, Christian Nabe, Georgios Papaefthymiou, Christoph Weber, and Hannes Weigt (2013). "Renewable electric energy integration: Quantifying the value of design of markets for international transmission capacity.” Energy Economics 40: 760-772. dx.doi.org/ 10.1016/j.eneco.2013.09.004 
Oggioni, Giorgia, and Yves Smeers (2012). "Degrees of Coordination in Market Coupling and CounterTrading.” The Energy Journal 33(3): 39-90. dx.doi.org/ 10.5547/01956574.33.3.3

Oggioni, Giorgia, and Yves Smeers (2013). "Market failures of Market Coupling and counter-trading in Europe: An illustrative model based discussion." Energy Economics 35: 74-87. dx.doi.org/ 10.1016/j.eneco.2011.11.018

Oggioni, Giorgia, Yyes Smeers, Elisabetta Allevi, and Siegfried Schaible (2012). “A Generalized Nash Equilibrium Model of Market Coupling in the European Power System.” Networks and Spatial Economics 12(4): 503-560. dx.doi.org/ 10.1007/s11067-011-9166-7

TenneT (2015). Network Figures. http://www.tennettso.de/site/en/Transparency/publications/networkfigures/overview, accessed August 31, 2015.

THEMA Consulting Group (2013). Loop flows - Final advice. Prepared for The European Commission. THEMA Report 2013-36.

https://ec.europa.eu/energy/sites/ener/files/documents/201310_loop-flows_study.pdf, accessed August 31, 2015.

TransnetBW (2015). Key Figures. https://www.transnetbw.com/en/key-figures, accessed August 31, 2015. 